\title{
ELEMENTARY PROOF OF HU'S THEOREM ON ISOTONE MAPPINGS
}

\author{
N. C. HSU
}

1. Introduction. Let $E$ be a partially ordered set of finite order, and let $m, n$ be two natural numbers. We pose the following questions: Find a necessary and sufficient condition under which there exists a mapping $f$ of $E$ into a linearly ordered set $L=\{1<2<\cdots<t\}$ such that

(a) $f$ is strictly isotone in the sense that $a<b$ in $E$ implies that $f(a)<f(b)$ in $L$.

(b) The cardinal number of $f^{-1}(n)$ is not greater than $m$ for every $n \in L$.

For the case where $E$ satisfies the condition that every element of $E$ is covered by at most a single element, a simple and elegant answer was given by $\mathrm{Hu}$ [2]. His proof is, however, far from simple. The purpose of this note is to provide a much simpler and more transparent proof of his theorem.

2. Preliminaries. Let $E$ be a partially ordered set of finite order. We define the height of an element in $E$ and the height of $E$ in a usual way (see, e.g. [1]). By the depth of an element $x$ in $E$, we mean the height of the element $\hat{x}$ in the dual $\hat{E}$ of $E$. By $h(E)$, we denote 1 plus the height of $E$. By $E_{i}$ and $E^{j}$, we denote the set of all elements of depth $i-1$ in $E$ and the set of all elements of height $j-1$ in $E$, respectively. For example, $E_{h(E)}$ is the set of all elements of maximum depth, and $E^{1}$ is the set of all minimal elements. Evidently, $E_{h(E)} \subseteq E^{1}$ is valid. We denote $E_{i} \cap E^{i}$ by $E_{i}^{j}$, and the cardinal number of $E$ by $|E|$. Finally, we put

$w_{i}(E)=\left|E_{h(E)}\right|+\left|E_{h(E)-1}\right|+\left|E_{h(E)-2}\right|+\cdots+\left|E_{h(E)+1-i}\right|$, for $i=1,2,3, \cdots, h(E)$. What makes our proof so simple is the following

Definition. Let $m$ and $t$ be two positive integers. $E$ is called $(m, t)$ bounded if and only if the following inequalities are satisfied:

$$
w_{i}(E) \leqq(i+t-h(E)) m, \quad \text { for } i=1,2,3, \cdots, h(E) .
$$

Our goal is to give a proof of

Theorem [2, p. 847]. Let E be a partially ordered set of finite order

Received by the editors May 25, 1965. 
in which every element is covered by at most a single element. Then, $E$ is $(m, t)$ bounded if and only if there exists a strictly isotone mapping $f$ of $E$ into the linearly ordered set $L=\{1<2<\cdots<t\}$ such that the cardinal number of $f^{-1}(n)$ is not greater than $m$ for every $n \in L$.

3. Proof of the theorem. We begin with a constructive proof of

Lemma 1. If a partially ordered set $E$ is $(m, t)$ bounded and also satisfies the condition

(0) $\left|E_{i}\right|>m$ for some $i$ implies that for the same $i$

$$
\left|E_{i}^{1}\right|+\left|E_{i+1}^{1}\right|+\left|E_{i+2}^{1}\right|+\cdots+\left|E_{h(E)}^{1}\right| \geqq m,
$$

then there exists a subset $F \subseteq E$ such that

(1) $|E-F| \leqq m$,

(2) either $E-F \subseteq E_{1}$ or $E-F \subseteq E^{1}$, and

(3) $F$ is $(m, t-1)$ bounded.

Proof. In case $\left|E_{1}\right| \leqq m$, let $F=E-E_{1}$. Then $h(F)=h(E)-1$ and

$$
\begin{aligned}
w_{i}(F) & =w_{i}(E) \\
& \leqq(i+t-h(E)) m \\
& =(i+t-1-h(F)) m,
\end{aligned}
$$

for $i=1,2,3, \cdots, h(F)$, and all three requirements are satisfied. In case $\left|E_{1}\right|>m$, we have $\left|E^{1}\right| \geqq m$ by the condition (0), and two subcases are conceivable; either $\left|E_{h(E)}^{1}\right|>m$ or $\left|E_{h(E)}^{1}\right| \leqq m$. If $\left|E_{h(E)}^{1}\right|$ $>m$, let $F=E-G$ where $G$ is a set of $m$ elements taken arbitrarily

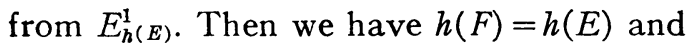

$$
\begin{aligned}
w_{i}(F) & =w_{i}(E)-m \\
& \leqq(i+t-h(E)-1) m \\
& =(i+t-1-h(F)) m,
\end{aligned}
$$

for $i=1,2,3, \cdots, h(F)$, and all three requirements are satisfied. On the other hand, if $\left|E_{h(E)}^{1}\right| \leqq m$, let $i_{0}$ be the integer satisfying both

$$
\left|E_{i_{0}}^{1}\right|+\left|E_{i_{0}+1}^{1}\right|+\left|E_{i_{0}+2}^{1}\right|+\cdots+\left|E_{h(E)}^{1}\right| \geqq m
$$

and

$$
\left|E_{i_{0}+1}^{1}\right|+\left|E_{i_{0}+2}^{1}\right|+\cdots+\left|E_{h(E)}^{1}\right|<m . ~ .
$$

Needless to say, we have $i_{0}=h(E)$ when $\left|E_{h(E)}^{\mathbf{1}}\right|=m$. Now, let 


$$
F=E-\left(E_{i_{0}+1}^{1} \cup E_{i_{0}+2}^{1} \cup \cdots \cup E_{h(E)}^{1} \cup G\right),
$$

where $G$ is a set of $m-\left(\left|E_{i_{0}+1}^{1}\right|+\left|E_{i_{0}+2}^{1}\right|+\cdots+\left|E_{b(E)}^{1}\right|\right)$ elements taken arbitrarily from $E_{i_{0}}^{1}$. Then $h(F)=h(E)-1$ and for $i=h(E)$ $-i_{0}+1, h(E)-i_{0}+2, h(E)-i_{0}+3, \cdots, h(F)$, we have

$$
\begin{aligned}
w_{i}(F) & =w_{i+1}(E)-m \\
& \leqq(i+1+t-h(E)-1) m \\
& =(i+t-1-h(F)) m .
\end{aligned}
$$

It remains to show that

$$
w_{i}(F) \leqq(i+t-1-h(F)) m
$$

for $i=1,2,3, \cdots, h(E)-i_{0}$. Suppose on the contrary that there exists a $j_{0}, 1 \leqq j_{0} \leqq h(E)-i_{0}$, such that

$$
\begin{aligned}
w_{j_{0}}(F) & >\left(j_{0}+t-1-h(F)\right) m \\
& =\left(j_{0}+t-h(E)\right) m .
\end{aligned}
$$

Assume that $j_{0}$ is the smallest integer having this property. Then

$$
\left|F_{h(B)+1-j_{0}}\right|=\left|F_{h(E)-j_{0}}\right|>m
$$

from which we have $\left|E_{h(E)-j_{0}}\right|>m$ and therefore by the condition (0),

$$
\left|E_{h(E)-j_{0}}^{1}\right|+\left|E_{h(E)-j_{0}+1}^{1}\right|+\cdots+\left|E_{h(E)}^{1}\right| \geqq m .
$$

Hence $h(E)-j_{0} \leqq i_{0}$ which implies $i_{0}=h(E)-j_{0}$. Consequently,

$$
\begin{aligned}
w_{j_{0}+1}(E) & =w_{j_{0}}(F)+m \\
& >\left(j_{0}+1+t-h(E)\right) m,
\end{aligned}
$$

contrary to the assumption that $E$ is $(m, t)$ bounded.

Lemma 2 [2, p. 844]. Let E be a partially ordered set of finite order which is not $(m, t)$ bounded. Then, there does not exist a strictly isotone mapping $f$ of $E$ into the linearly ordered set $L=\{1<2<\cdots<t\}$ such that the cardinal number of $f^{-1}(n)$ is not greater than $m$ for every $n \in L$.

Proof. If $E$ is not $(m, t)$ bounded, there exists an $i$ such that

$$
w_{h(E)-i+1}>(t+1-i) m .
$$

Suppose on the contrary that there exists a mapping $f$ described in the statement. Then, since each of $f^{-1}(1), f^{-1}(2), \cdots, f^{-1}(t+1-i)$ consists of at most $m$ elements, there must exist an $x_{i} \in E_{i}$ such that 
$f\left(x_{i}\right)>t+1-i$. Since $f$ is strictly isotone, there must exist an $x_{1} \in E_{1}$ such that $f\left(x_{1}\right)>t$, contrary to the assumption that $f(x) \leqq t$ for every $x \in E$.

Proof of The theorem. Suppose that $E$ is $(m, t)$ bounded. Since every element of $E$ is covered by at most a single element, the condition (0) in Lemma 1 is satisfied by every subset of $E$, and a desired mapping can be constructed, by finite induction, by means of Lemma 1. The converse is an immediate consequence of Lemma 2.

UNSOLVED PROBLEM. Let $m$ and $t$ be two positive integers. Find a necessary and sufficient condition under which there exists a strictly isotone mapping $f$ of a more general partially ordered set $E$ in to the linearly ordered set $L=\{1<2<\cdots<t\}$ such that the cardinal number of $f^{-1}(n)$ is not greater than $m$ for every $n \in L$.

The author is indebted to T. C. Hu for personal communication.

\section{REFERENCES}

1. G. Birkhoff, Lattice theory, Amer. Math. Soc. Colloq. Publ. Vol. 25., Amer. Math. Soc., Providence, R. I., 1948.

2. T. C. Hu, Parallel sequencing and assembly line problems, Operations Res. 9 (1961), 841-848.

State University of New York at Buffalo 